\title{
Entretien avec Freddy Tsimba
}

\section{Thérèse De Raedt}

URL : http://journals.openedition.org/coma/611

DOI : 10.4000/coma.611

ISSN : 2275-1742

\section{Éditeur}

Institut des textes \& manuscrits modernes (ITEM)

\section{Référence électronique}

Thérèse De Raedt, "Entretien avec Freddy Tsimba », Continents manuscrits [En ligne], 4 | 2015, mis en ligne le 15 octobre 2015, consulté le 16 avril 2021. URL : http://journals.openedition.org/coma/611 ; DOl : https://doi.org/10.4000/coma.611

Ce document a été généré automatiquement le 16 avril 2021.

\section{(c) (i) ()}

Continents manuscrits - Génétique des textes littéraires - Afrique, Caraîbe, dispora est mis à disposition selon les termes de la licence Creative Commons Attribution - Pas d'Utilisation Commerciale - Pas de Modification 4.0 International. 


\title{
Entretien avec Freddy Tsimba
}

\author{
Thérèse De Raedt
}

\section{Introduction}

1 Né en 1967 d'une très jeune maman et d'un père polygame, Freddy Tsimba passe sa toute petite enfance chez ses grands-parents près des chutes Inga. Il parle kikongo, ses parents étant kongo du Manyanga. Il grandit à Kinshasa dans le quartier Matongé où il habite toujours. ${ }^{1}$

2 En 1989 il obtient son diplôme en arts plastiques, section sculpture monumentale de l'Académie des Beaux-Arts de Kinshasa. Ensuite il passe six ans chez les maîtres fondeurs car, selon lui, ils sont les détenteurs « de la continuité culturelle ». ${ }^{2}$ Dès le début des années 1990, il se libère des conventions académiques acquises à l'Académie des Beaux-Arts et crée des sculptures en bronze filiformes, inspirées de lianes. Elles sont contorsionnées et entrelacées pour montrer la souffrance sociale mais aussi pour faire ressortir l'union entre les hommes. ${ }^{3}$ 
Fig. 1 : "Lutte pour la survie" 2001

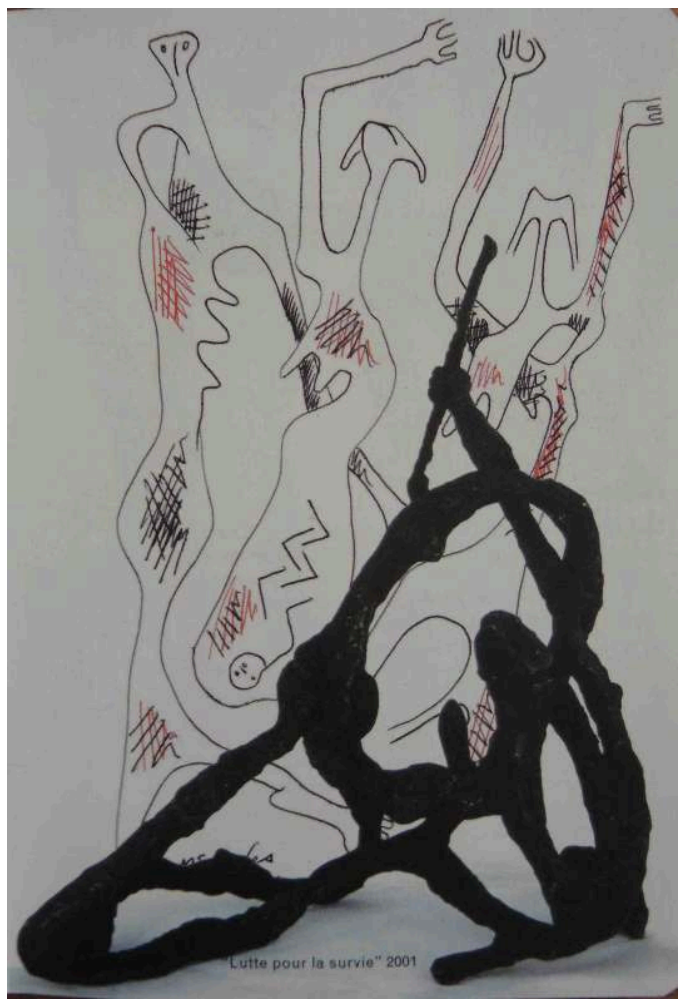

Repris du livre Freddy Tsimba, sculpteur, texte de Yoka Lye, Montreuil, Éditions de l'oeil, 2002, p. 13 Guillaume Pierre et Dieudonné Ndula.

Depuis 1998 il récupère des matériaux en rue (tels des cuillères, fourchettes, capsules de bière, clés etc.) et sur les champs de bataille (balles, douilles, cartouches, restes d'obus). Il les fond et/ou les rassemble en leur donnant une forme humaine, généralement féminine. (Les femmes qu'il réalise sont souvent enceintes.) Ainsi transforme-t-il des "déchets ", des choses dont on s'est servi et puis qu'on a jetées, en œuvres d'art au symbolisme puissant, et recycle-t-il des objets inertes en leur donnant un pouvoir humanisant. En 2001 son œuvre Victime malgré elle, qui représente une femme assise dont la tête est formée par une cuillère pour symboliser la famine, reçoit la médaille d'argent à la quatrième édition des Jeux de la Francophonie qui avaient eu lieu à Ottawa. ${ }^{4}$ Cette reconnaissance lui confirme qu'à travers son art de refonte, il a une mission dans la vie. Artiste engagé il se veut solidaire avec la souffrance non seulement en RDC mais dans le monde entier. En la dénonçant il veut aussi la transcender en montrant la force et l'énergie qu'offre la vie.

Fig. 2 : "Victime malgré elle", œuvre qui a remporté la médaille d'argent aux Jeux de la Francophonie à Ottawa en 2001 


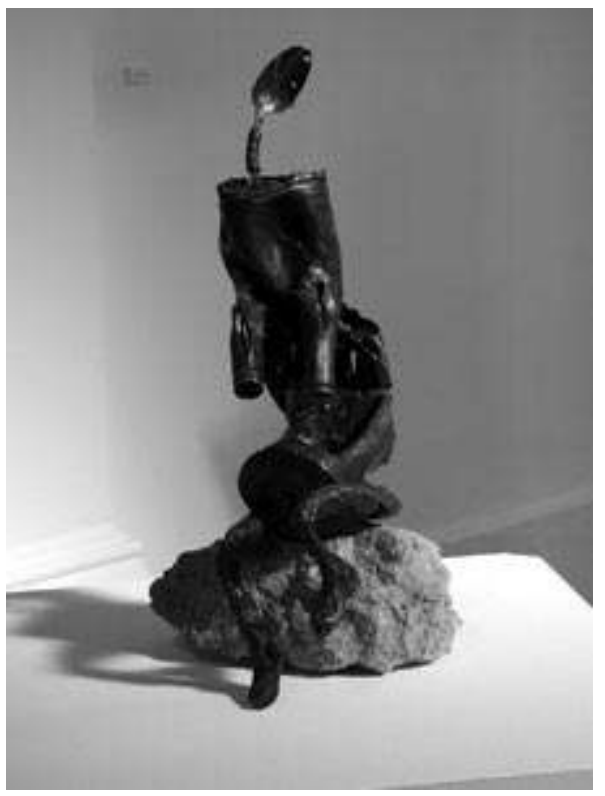

Repris du site: https://freddytsimba.wordpress.com/2012/03/19/sculptures/\#jp-carousel-292 https://freddytsimba.wordpress.com/2012/03/19/sculptures/\#jp-carousel-292

4 Vers les années 2010 il crée en rue dans son quartier sa première maison machette faite de 999 machettes. Il réalise aussi des œuvres à partir de crânes de singes (récoltés auprès de gens de la région de l'Équateur pour qui les singes constituent une nourriture) pour fustiger la déforestation et la mise en péril de l'environnement. ${ }^{5} \mathrm{En}$ témoignant ainsi de l'histoire immédiate, il veut contribuer à un effort de mémoire et laisser des traces.

5 Freddy Tsimba a participé à une trentaine d'expositions dans le monde entier (Afrique du Sud, Algérie, Allemagne, Belgique, Benin, Canada, États-Unis, France, Haïti, Maroc et Sénégal) et a été invité à participer à plusieurs résidences d'artistes comme en 2007 à la Fondation Jean-Paul Blachère. En 2002 il a reçu en RDC la médaille des Arts, des Sciences et des Lettres et en 2007 le prix du Meilleur Artiste Plasticien de la RDC.

6 Fin septembre 2007 la commune d'Ixelles à Bruxelles inaugure sa sculpture monumentale intitulée Au-delà de l'espoir ou, comme Tsimba aime aussi l'appeler, Après l'espoir : la vie. ${ }^{6}$ Conçue avec des douilles, elle représente une femme tenant un enfant, le regard perdu dans le ciel. J'ai rencontré Freddy Tsimba à Bruxelles dans le quartier de Matongé, pas loin de cette œuvre, le 9 août 2014.

Fig. 3 a : "Au delà de l'Espoir" avec Freddy Tsimba 


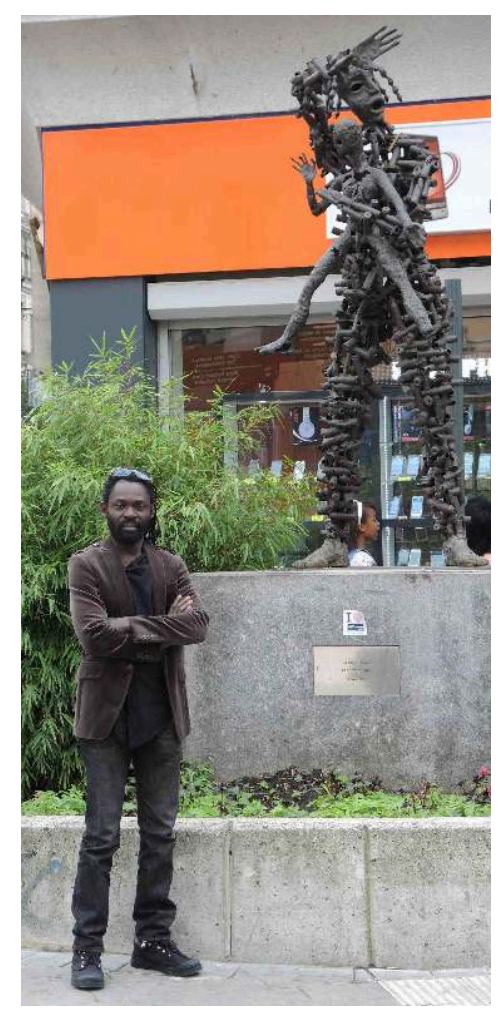

Photo prise le 9 août 2014 à Ixelles (quartier Matongé)

Thérèse De Raedt

7 L'entretien porte sur ses dernières œuvres et sur son cheminement artistique.

\section{Entretien}

Q. Pourriez-vous vous présenter et nous dire comment vous êtes arrivé aux arts plastiques?

R. Mon nom d'artiste est Freddy Tsimba mais mon long nom est Freddy Bienvenu Tsimba Mavambu. ${ }^{7}$ Je suis né à Kinshasa un certain 22 août 1967. C'était l'époque où le pays avait changé de régime avec Mobutu. ${ }^{8}$ Donc on peut dire que je suis, ainsi que toute ma génération, un des enfants de ce monsieur-là. On a vécu son pouvoir d'une trentaine d'années mais on a aussi vécu plein de choses car on habitait Matongé. Matongé est le quartier fort, chargé du pays. C'est dans ce quartier qu'en 1969 des étudiants ont été massacrés. Matongé est aussi le lieu où en 1992 des chrétiens ont été tués. ${ }^{9}$ C'était aussi à Matongé que les gens se sont rassemblés pour demander l'indépendance du pays. Matongé c'est comme un bastion fort de la résistance. Mais c'est aussi comme un pays. C'est une référence. Il y a des Matongé partout : à Bruxelles, en Ouganda etc. Matongé est notre source.

10 Je viens d'une famille de 15 enfants. Être 15 faisait beaucoup de bruit! J'ai hérité, je crois, de l'état de mon père qui, polygame, m'a transmis la notion de partage ; c'est à dire que puisque nous étions 15 enfants nous devions être soudés (et même si nous étions nés de mères différentes). Mon père tenait à l'équilibre de nous tous. Comme j'étais l'aîné de ma maman, j'ai dû apprendre qu'il y avait des différences pour ensuite pouvoir les négocier. 
Comme j'aimais beaucoup dessiner on m'a orienté vers ce que je suis maintenant. Maintenant je me décris comme plasticien.

Q. Vouliez-vous être plasticien depuis votre plus jeune âge?

R. Oui. Depuis toujours. À l'âge de 6 ans, je faisais des voitures avec du fil de fer pour mes amis. Je ne demandais pas grand' chose - l'équivalent d'un euro ou quelques centimes mais quand j'en faisais 5 ou 6 cela me faisait 3 ou 4 euros peut-être. Tout l'argent que je gagnais je l'employais pour acheter des choses comme des sousvêtements. Cela fait rire mais cela me permettait d'être un peu à l'aise. Cela m'a beaucoup aidé.

Puisque je n'avais pas de moyens pour acheter des crayons ou d'autre matériel pour dessiner mais que j'avais devant moi les locaux de la parcelle, je dessinais partout. Quand quelqu'un piétinais mes dessins je pleurais car c'était comme si quelqu'un venait de tuer quelque chose.

Q. Vous dessiniez par terre?

R. C'est ça, sur de l'argile mélangée. Le sol était noir. Moi je trouvais cela magnifique. C'est comme si je faisais des graffitis. Je dessinais des scènes de vie, des scènes d'amour, des scènes de mon quartier. Quand nous allions dehors il y avait de l'animation dans les rues et, sans le savoir, j'ai internalisé ces bruits, ces morceaux de vie, ces bouts de phrases. Je pense que cela fait aussi partie de mon héritage et que cela m'a forgé.

Q. Vous avez été à l'école des Beaux-Arts à Kinshasa. ${ }^{10}$ Que s'est-il passé entre temps ?

R. Quand j'ai eu terminé mes six années de primaire, on nous a demandé ce que nous voulions faire. ${ }^{11}$ Moi j'avais envie depuis toujours d'aller aux Beaux-Arts mais quand je suis arrivé aux Beaux-Arts tout était plein. Alors j'ai pensé au journalisme car j'aimais bien voir les gens parler à la télé. Cela me fascinait. On pensait que ces gens étaient intelligents pour parler si bien or il y avait des moniteurs mais nous ne le savions pas à l'époque. Je voulais faire comme eux. Mais mon malheur est que j'ai un cheveu sur la langue. Alors, j'ai fait deux années dans une école scientifique mais je ne voulais pas faire des mathématiques, des sciences exactes. Je voulais faire autre chose. Je voulais faire des choses qui me faisaient voyager, faire des choses qui transcendaient le temps et qui laissaient des traces.

Q. En somme vous vouliez créer?

R. Oui je voulais créer. Et dieu merci une de mes sœurs était amoureuse d'un homme qui travaillait aux Beaux-Arts. Et un jour il est venu à la maison et il m'a vu dessiner. Il a demandé si c'était moi qui avais fait tout cela. Et les autres ont répondu que depuis que j'étais petit ils ne m'avaient connu qu'en train de dessiner. Alors ce monsieur a demandé qu'on m'appelle. Je lui ai montré ce que j'avais déjà dessiné sur des bouts de papier et sur tout ce que je trouvais. C'était la première fois que quelqu'un voulait voir ce que j'avais fait. Il a dit: "Ah toi tu dessines bien. Tu es à l'école des Beaux-Arts?» Quand je lui ai dit "non " il m'a dit qu'il était de là et m'a demandé de lui donner quelques dessins. J'ai dessiné deux ou trois fleurs derrière l'un d'eux avec un stylo. J'étais très concentré ! Il m'a dit qu'il ne pouvait rien me promettre mais qu'on verrait. Ainsi est arrivé aux Beaux-Arts un de mes dessins. Sur les 500 jeunes qui voulaient s'inscrire, ils ont pris 50 personnes. Et j'étais parmi ces 50 . J'étais aux anges. Je suis entré aux Beaux-Arts en $4^{\text {ème }}{ }^{12}$ 

Je me disais "ils sont déjà en avance ». Moi je ne connaissais pas cela mais j'ai fait semblant. J'ai fait comme eux car je ne voulais pas être agressé dans mon amourpropre. Après j'ai aussi compris qu'il ne fallait pas seulement dessiner ce que l'on voyait. J'ai appris qu'il fallait dessiner ce que l'on voyait et comment son corps réagissait par rapport à cela. Au début c'était compliqué et puis un jour j'ai vu mon dessin affiché aux valves. ${ }^{13}$ Pour moi c'était la consécration. Je suis allé chez mon père et lui ai dit : « cette fois-ci je crois que j'ai ma voie.» tu as ton bac ou chez nous ce qu'on appelle le diplôme d'état, tu peux faire n'importe quoi. Tu peux aller sur le campus ou où tu veux. Moi je voulais continuer aux BeauxArts. Mais au départ on m'avait mis dans la section céramique. Pour moi faire de la céramique c'était comme si on me demandait de faire des marmites. Je ne voulais pas cela. J'en faisais des cauchemars. Mon père était tellement de mon côté qu'il est allé voir le directeur et a dit : «Mon fils fait des cauchemars, je crois qu'il faut le changer.» Mon père a obtenu que l'on me change et j'ai fait la sculpture pendant trois ans de supérieur. Mais je crois que j'ai plus appris après l'école des Beaux-Arts que j'ai terminée en 1989.

traces et pour cela je devais travailler sur le terrain. Car, qu'on le veuille ou non, l'art c'est aussi une sorte d'apostolat. On doit assumer si ça marche ou si cela ne marche pas. On va mais on ne sait pas où l'on va atterrir. Quand je voulais me démarquer, par ce que je faisais, des aînés à l'époque, je suis allé chez des artisans pour apprendre la technique, les rudiments du métier. Je suis allé chez des bijoutiers, des fondeurs, des forgerons pour apprendre comment manipuler l'acier, la matière, le cuivre, le bronze etc. J'ai passé six ans dans leurs ateliers. Mais en même temps je faisais aussi mes œuvres qui commençaient à se démarquer. Et chaque fois que je venais à exposer les gens se disaient: «mais qu'est-ce que tu fais toi ? C'est quoi ça? Avec cela tu ne vas jamais grossir! » Et en effet je n'ai jamais grossi ! C'était une façon pour eux de me dire que tu fais des choses mais pour nous ce n'est pas de l'art. 
R. Non. Par exemple il y avait des œuvres comme celle que j'ai nommée « Femme aux deux bassins vides ». C'est une femme qui a perdu certains de ses enfants et qui a une bassine sur la tête mais qui est vide. J'ai employé des codes comme cela où les hommes et les femmes représentent une certaine souffrance. Quelqu'un à l'époque a écrit : "Freddy Tsimba: sculpteur de la souffrance ». Les gens ont vu dès cette époque la souffrance dans mon travail et cette appellation m'est restée collée à la peau.

J'ai vécu dans une famille où il y avait pas mal de problèmes ou, peut-être devrais-je plutôt dire, pas mal de frustrations car on était 15 enfants. Nous craignions notre père qui souhaitait que nous réussissions. Lui n'avait pas fait de grandes études mais avait pu arriver à vivre sa vie tranquille, comme il faut : avoir une maison, un terrain.

Q. Votre père quelles études avait-il faites?

R. Mon père avait fait les études normales. À l'époque cela n'était pas grand-chose mais il était intelligent. C'est lui qui fixait les prix des denrées alimentaires à Kinshasa. Il était un intellectuel aussi. Mais jusqu'à ce qu'il meure personne n'a jamais pu vraiment s'épanouir. Quand je vois mes frères et mes sœurs, je constate qu'il y a quand même eu une certaine crainte. En ce qui me concerne, il a vu que son fils a pu émerger.

Q. Dès le départ avez-vous mélangé les matériaux riches (comme le bronze, l'argent et l'or) et les matériaux dits " pauvres »?

R. Bon, non. Quand j'ai commencé à fondre le métal j'ai fondu du cuivre. J'en faisais du bronze que je patinais en noir. Les gens associaient parfois mes œuvres à celles de Giacometti mais moi j'ai dit que cela n'était pas vrai. Giacometti s'est inspiré des Dogons donc il ne faut pas récupérer ce qui est à lui. Donc j'ai dit aux gens : « non, moi j'ai fait mon travail. Ce n'est pas Giacometti. C'est moi. »

Et puis, j'ai commencé à un peu travailler le fer. Du fer que j'ai découpé. J'allais chercher des portes, du fer qui a vécu. Je demandais aux gens si je ne pouvais pas remplacer leur porte, je leur en donnais une autre et prenais l'ancienne et en découpais des silhouettes. Pour moi c'était pour dire: les portes sont comme des personnages. C'était aussi une manière de changer l'image des portes et tout cela. J'ai fait plusieurs œuvres dans ce sens là. Au fur et à mesure - car je ne suis jamais satisfait - j'ai regardé autour de moi pour créer autre chose. Et là j'ai commencé à faire des œuvres avec des personnages qui marchaient. À Kinshasa les gens marchent des kilomètres et des kilomètres. Alors je me suis dit: "pourquoi ne pas faire des œuvres avec des piétons mais des piétons aux pieds fatigués?» Ces œuvres je les ai donc appelées Piétons fatigués.

\section{Q. En quelle matière?}

R. En bronze patiné, noir comme cela. J'ai fait plein de personnages qui, fatigués, marchent. (Je n'ai plus beaucoup de photos de ces œuvres.) Et dans cet élan là j'ai fait aussi des couples: des couples avec un enfant mort, des gens qui marchent avec un enfant malade. ${ }^{14}$

Q. Vous réalisez surtout des sculptures?

R. J'aime bien la nature et la sculpture est plus proche de la nature. La sculpture est aussi plus proche de l'homme. La sculpture prend l'espace. Elle est là. Elle vous envahit. Elle contraint l'homme à vivre avec elle. Si tu t'en vas, tu la laisses là. C'est ça que j'ai voulu faire. En ce qui concerne la peinture, je dis «j'ai peint». Ce n'est pas quelque chose que je mets en relief. 
Q. Pourriez-vous nous parler plus de vos œuvres réalisées avec des douilles? Comment avez-vous trouvé les douilles?

R. J'ai récupéré des douilles dans des lieux de guerre pour en faire des œuvres. Certaines viennent du Bas-Congo, d'autres de Kinshasa (suite à l'explosion du dépôt de munition à l'aéroport Ndjili en avril 2000) ou de Kisangani. C'était dangereux de ramasser des douilles à Kisangani mais j'ai pris le risque. Maintenant je ne le ferais plus car je suis un peu connu. ${ }^{15}$ J'ai aussi des douilles de Haïti que j'ai intégrées dans ces œuvres et même des douilles que j'ai eues en Normandie. Pas beaucoup mais ce sont des douilles que j'ai ramenées au pays. J'ai aussi pris des douilles à Soweto. Donc ce sont des douilles que j'ai prises de partout.

Alors pourquoi des douilles? Les gens et certains critiques disent que c'est par rapport aux guerres. Moi je dis : «OK, mais c'est aussi le style de mon travail.» Je dis par rapport à cela : la douille c'est aussi comme un oiseau. Sauf que l'oiseau ne tue pas. La douille qui est fabriquée à Moscou par exemple peut arriver en RDC sans problème. Estce qu'il y a des douilles sans papier ? Non. La douille arrive en RDC comme cela. En plus la douille est exportée et est suivie par des gens. Moi pour que je vienne ici [ = en Belgique] il faut des papiers, il faut être invité etc. et il faut avoir un compte bancaire ou vingt mille dollars. Donc je me suis dit que j'allais faire des œuvres avec des douilles car ainsi les œuvres allaient bouger et elles allaient faire le voyage dans le sens contraire. C'est ça mon message. Elles bougent, elles peuvent revenir. Elles peuvent être mises dans des expositions, dans les plus beaux lieux du coin, dans des musées. Mais ce sont aussi des douilles qui ont tué des gens. Il faut rendre hommage à ces gens. Ces milliers de gens qui sont morts et pas qu'en RDC.

Fig. 3 b : "Au-delà de l'espoir" Congo 2007

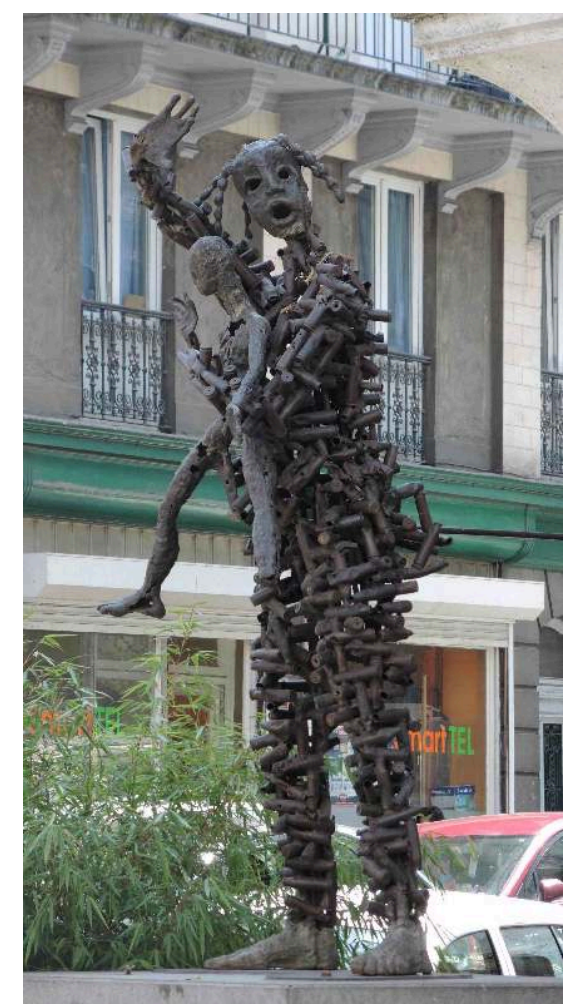

Photo prise le 9 août 2014 à Ixelles (quartier Matongé)

Thérèse De Raedt

Continents manuscrits, 4 | 2015 
R. Je récupère à Kinshasa des cuillères que les gens ont jetées. Les gens de la rue m'aident à en récupérer. Et ça, ça m'aide car ainsi je donne du boulot aux autres. Je ne suis pas millionnaire mais le peu que je gagne je peux le partager avec les enfants de la rue. Il y en a beaucoup, ils me connaissent et ils m'appellent: "rasta papa Freddy l'artiste. » Je contribue ainsi un petit peu à quelque chose, à l'essor de quelque chose. Ça peut être petit mais au moins on est là présent. On vit là.

Fig. 4 : "Homme caméra" 2012

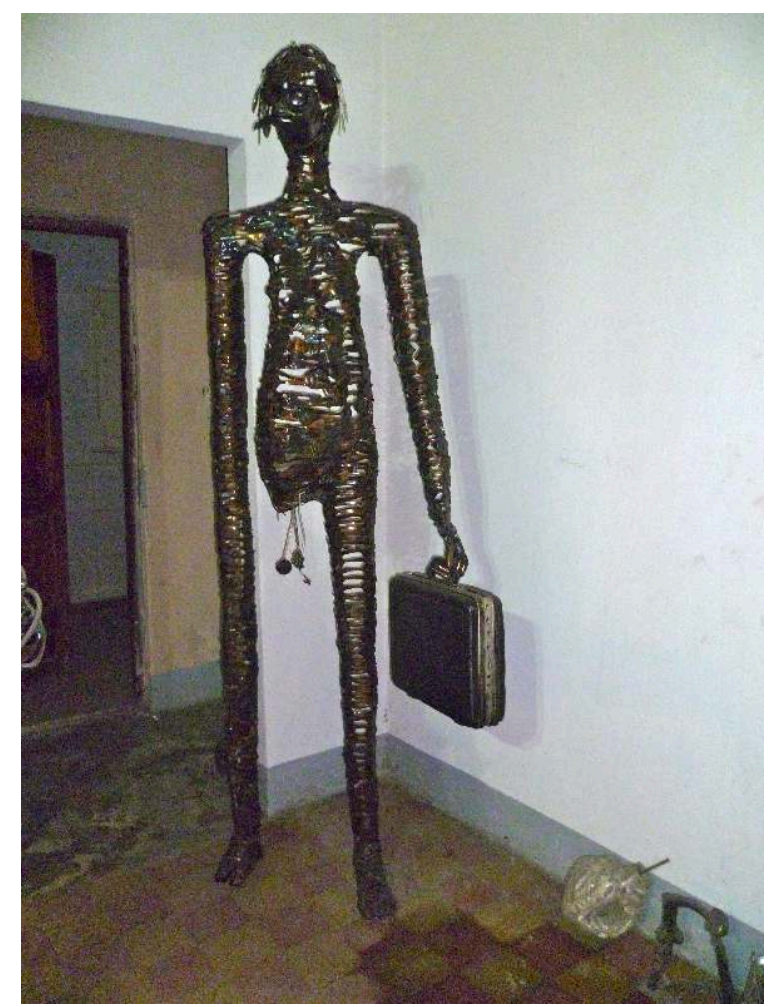

PHOTO PRISE LE 4 JUILLET 2012 DANS L'ATELIER DE FREDDY TSIMBA À KINSHASA THÉRÈSE DE RAEDT

Moi je trouve dans mon quartier chaque jour quatre ou cinq cuillères sans chercher. Les enfants ils vont plus loin que moi et donc ils en trouvent plus. Chaque jour ils en 
trouvent entre 30 et 40 . En ce moment je suis en rupture de stock. Au retour je crois que je vais trouver 3000 cuillères. Il faut juste avoir le temps de patienter. cuillère dedans, il va courir pour prendre la cuillère car la cuillère signifie nourriture, signifie la vie. Avec mes œuvres je dénonce qu'il y a des problèmes de malnutrition car si je crée des corps en soudant des couverts, ça signifie qu'il y a un problème aussi, qu'on n'a pas les moyens d'en faire usage. Je dénonce ainsi les carences en nourriture sur terre. Cela m'a bouleversé de voir à la télé comment des gens peuvent perdre toute leur énergie et devenir comme rien du tout. Des gens qui doivent marcher et qui vont mourir parce qu'ils n'ont rien à manger. J'ai été particulièrement interpellé en voyant à la télévision une femme courir vers des sacs de nourriture qui étaient lancés par avion lors de la guerre en Somalie. Elle courait moins vite que les autres car elle portait un enfant.

Fig. 5 : Atelier de Freddy Tsimba

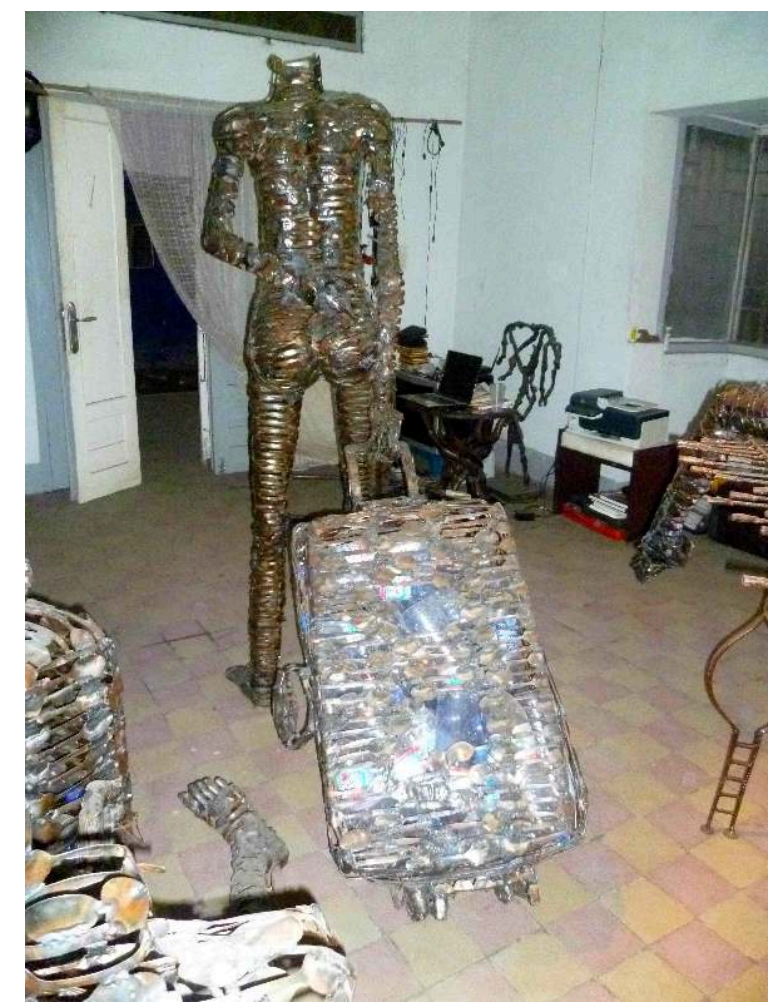

PHOTO PRISE LE 4 JUILLET 2012 DANS L'ATELIER DE FREDdY TSIMBA À KINSHASA THÉRÈSE DE RAEDT

Q. Les nouvelles vous inspirent-elles?

52 R. Je les écoute beaucoup mais je ne les réutilise pas directement dans mon travail. J'ai mon point de vue et je cherche les raisons. Je dis ce que je pense et je vais jusqu'au bout.

53 Je ne veux pas que mon œuvre soit une œuvre humanitaire. Je veux juste qu'elle soit un regard par rapport à la vie, de ce qui se passe autour de moi. Tant que l'homme vivra, il 
y aura de la souffrance. Moi je ne l'ai pas inventée mais par mon travail je veux que l'on en parle. C'est pour cela que je ne suis pas trop connu.

Q. Qu'en est-il pour les machettes? Comment les trouvez-vous?

R. Les machettes c'est vrai que je ne les ai jamais ramassées. On ne peut pas les ramasser. Ces machettes viennent de Chine. Je dois les commander. Ce sont des projets.

J'ai fait une résidence en Chine à Quanzou avec cinq Chinois et cinq Congolais. J'avais dit que je voulais faire une pagode chinoise avec des machettes. ${ }^{17}$ Le projet avait été accepté mais les machettes ne sont jamais arrivées. Donc l'œuvre n'a jamais été réalisée. Pourtant, j'avais commandé des machettes ... Finalement j'ai fait la maison à Kinshasa grâce à une amie qui voulait vraiment que je fasse l'œuvre et elle a mis le paquet: elle a acheté les machettes via les Libanais et c'est ainsi que j'ai fait l'œuvre à Kinshasa. Je voulais à tout prix montrer la violence urbaine. Mais la machette n'est pas seulement ce qui tue. C'est aussi la vie.

57 Q. La maison, c'est le foyer.

Fig. 6 a : Maison Machettes

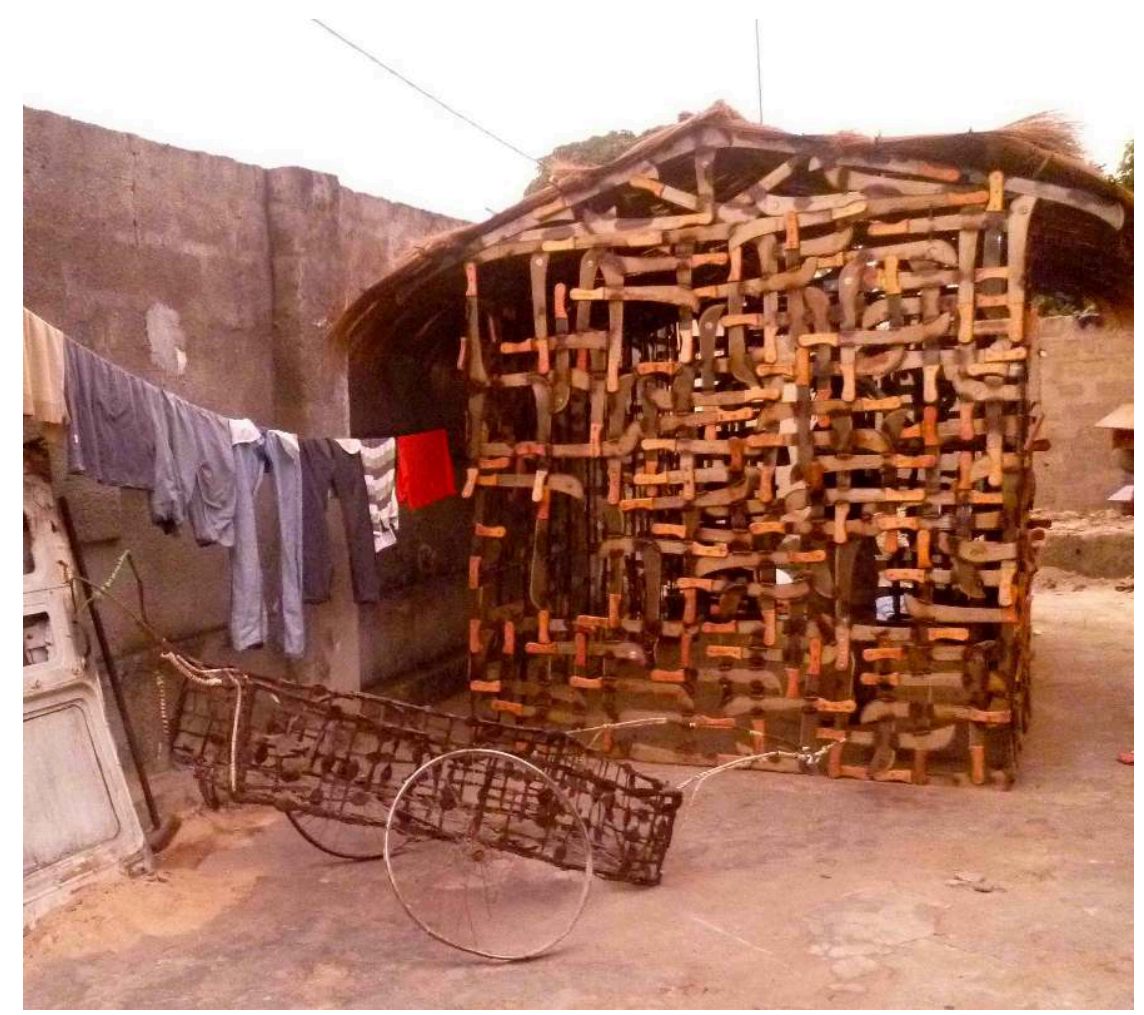

Photo prise le 4 juillet 2012 dans la concession de l'atelier de Freddy Tsimba à Kinshasa THÉRÈSE DE RAEDT 


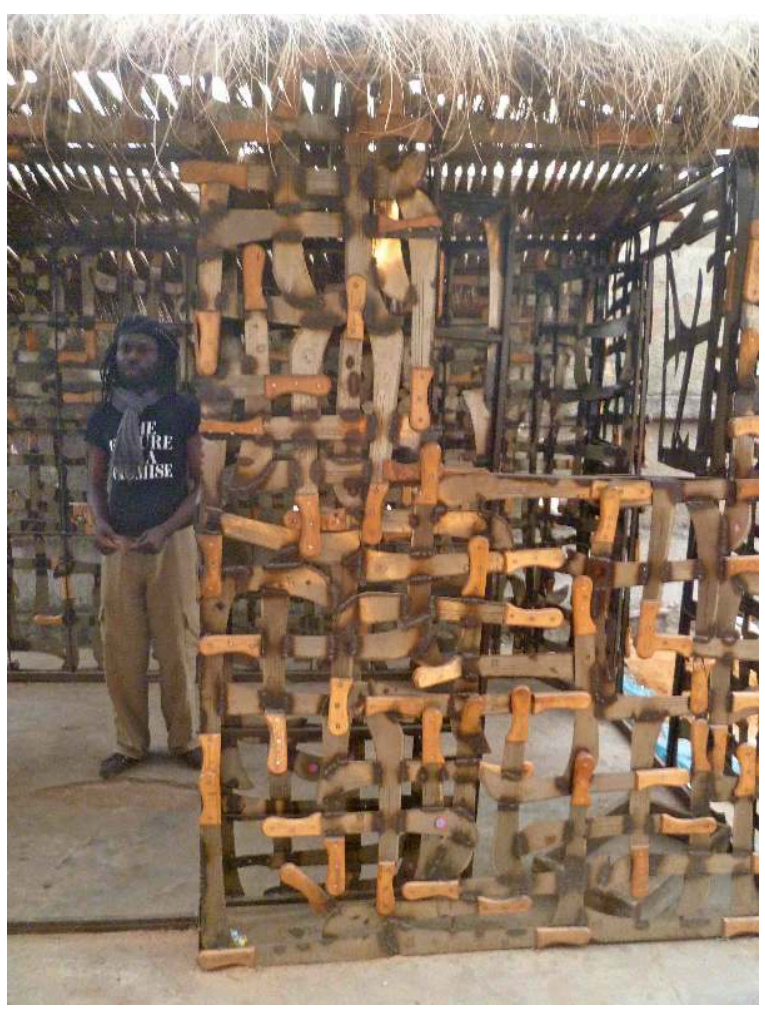

Photo prise le 4 juillet 2012 dans la concession de l'atelier de Freddy Tsimba à Kinshasa THÉRÈSE DE RAEDT

R. Voilà. C'est le lieu de l'éducation, de la procréation. C'est le lieu où le sens même est donné. Mais je me pose des questions : «Est-ce que nos maisons sont-elles maintenant des lieux où l'on donne l'éducation? » Quand je dis maison, cela peut être aussi un pays. Est-ce que l'on est plus en sécurité dans sa maison ou dehors? Ce sont des questions que je me pose. Mais quand je fais une maison en machettes, je ne vais pas donner de réponses. Je pose juste un acte et l'acte est de faire une maison machettes. Qu'est-ce qu'elle n'a pas fait la machette? Elle a fait plein de choses dans nos pays. Mais la machette au départ c'était pour défricher la terre et donc pour aider l'homme à vivre, à survivre, à vivre bien sur terre. Toutefois elle a aussi été détournée par l'homme car c'est l'homme qui l'a prise pour dire « ça maintenant, c'est pour ôter la vie, c'est pour faire du mal.» Quand je fais cette maison c'est pour dire : «La machette ce n'est pas pour tuer c'est pour construire.»Ce sont ça les mots. C'est pour cela que je voulais à tout prix que cette œuvre soit transportée dans les rues de Kinshasa, comme un cercueil pour extirper le mal. Les gens chantaient. Nous disons que la maison est quelque chose de fort mais voilà comment elle est: elle est faite de machettes, qui symbolisent la violence. ${ }^{18}$ Les deux mondes cohabitent. On ne peut pas éviter la violence. Partout où l'on va, il y en aura toujours.

J'ai déjà réalisé plusieurs maisons et j'adapte la maison par rapport au lieu où je suis. J'ai fait une maison à Abomey au Bénin,${ }^{19}$ une à Casablanca. ${ }^{20}$ À Ostende j'ai fait une case machette par rapport au lieu : Ostende avec la couronne royale. ${ }^{21}$ Léopold II était là. Les gens qui ont l'œil vont voir.

Q. Pourquoi une case à Ostende plutôt qu'une maison? 
61 R. La case c'est l'origine de l'homme que j'essaie de traduire à ma façon. On peut se poser la question: est-ce que l'homme a évolué ? Est-il différent de celui qui vivait avant? On ne saura jamais. Cette case nous parle à nous tous.

Q. Pourriez-vous nous parler de la genèse de votre dernière œuvre Centre fermé, rêve ouvert, qui représente des personnes les mains collées au mur dans une position humiliante?

R. Elle est née comment ? En 2011 j'avais un visa de travail pour Strasbourg. Je suis allé à Strasbourg pour travailler mon œuvre. J'y ai fait les six mois. Après je devais rentrer à Kinshasa puis devais revenir. Quand je suis revenu, j'avais un vol Kinshasa - Zaventem (en Belgique) pour, ensuite, de là, aller à Strasbourg. Mais j'ai été arrêté à Zaventem, à l'aéroport. On m'a dit que mon visa n'était plus valable et que j'étais irrégulier. Ensuite on m'a mis en tôle, dans le centre INAD [ = pour les personnes INADmissibles]. Je suis resté 10 jours dans ce lieu fermé. ${ }^{22}$

Q. Y avait-il d'autres personnes?

R. Oui. C'était le mois d'octobre. Ça je ne l'oublierai jamais. J'ai vu l'humiliation, les gens qui étaient là avec moi dans ce lieu pourri et ils attendaient comme moi. C'était vieux. Ça sentait mauvais. Les gens tombaient malades car on était dans des sortes de boxes avec des vitres. Nous voyions les avions atterrir et partir mais eux ne nous voyaient pas. Je me disais mais moi je ne peux quand même pas rester comme cela ici. On va montrer mon film à la télé. Je veux montrer aux autres mon travail. Même les gardiens ont vu que j'étais différent. Ils m'ont dit de demander l'asile politique. Mais moi je leur ai dit que je n'avais pas besoin de ça. J'ai dit «non ». Je voulais rentrer au pays car j'étais tellement mal dans ce lieu. À ce moment il y a aussi des gens qui ont commencé à réagir. ${ }^{23} \mathrm{Il}$ y a eu plus de 2000 personnes qui ont signé une pétition etc. Arrivé en RDC, mon passeport a été confisqué par les Congolais. Ils l'ont confisqué pendant un an. Pendant une année j'étais donc comme un oiseau dans une cage. J'étais bloqué et il m'était interdit de voyager. Pendant ce temps là, mon œuvre est née. Cette œuvre m'a soigné. Est-ce que j'aurais pu faire cette œuvre sans passer par là. Je ne sais pas. Fallaitil vraiment passer par là ? Mais au moins j'ai fait l'œuvre que j'ai intitulée : «Centre fermé, rêve ouvert ». Mon rêve est toujours ouvert et le restera.

Fig. 7 : "Centre fermé, Rêve ouvert" 2011 


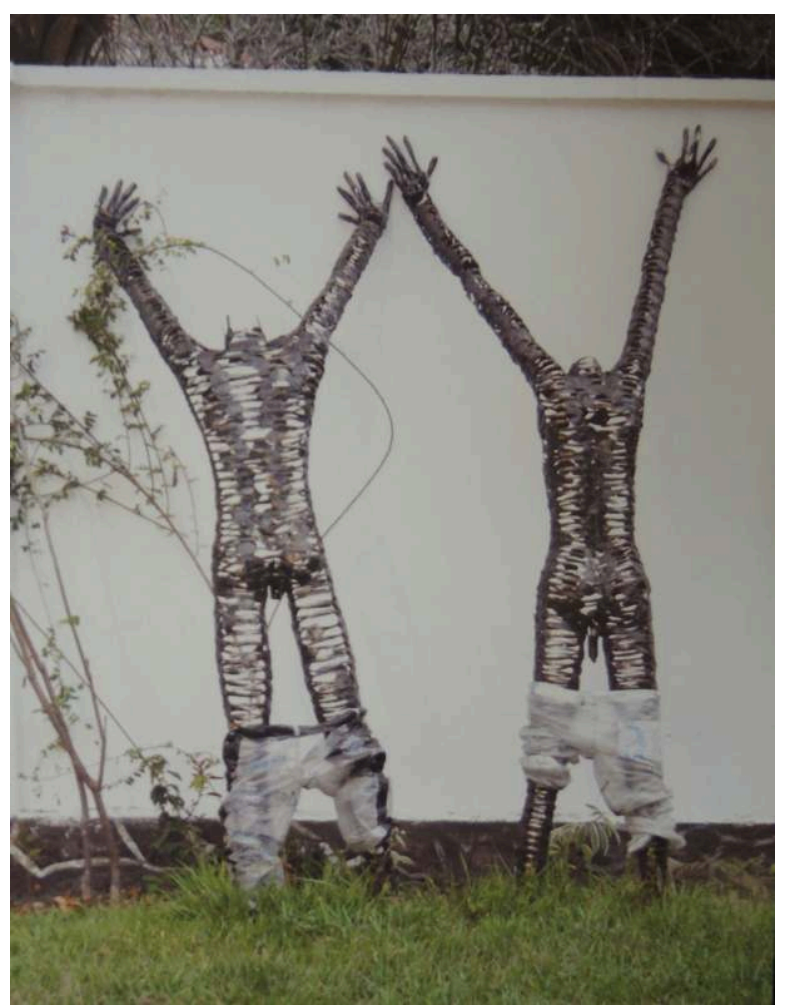

Repris du livre Freddy Tsimba, légendes et saisons de métal, texte de Vincent Lombume Kalimasi, Bruxelles, Le Cri édition, 2012, p. 13

CÉDRICK NZOLO NgAMOBU

main d'abord. Parce que je dis toujours qu'il n'y a qu'une race, la race humaine. Si elle ne mange pas, elle crève. Mon travail n'est pas un travail centré sur la guerre en RDC. Il est centré sur la violence, sur le vécu mais surtout aussi sur la vie. Quand j'ai fait ma maison machettes, les gens voulaient y donner un sens par rapport à la guerre. $\mathrm{Ce}$ n'est pas ça seulement. Je n'ai pas besoin de prendre un micro pour crier cela. 
veulent ou non les gens finiront toujours par reconnaître le bon travail. Les gens au fond d'eux-mêmes sont sincères et cette sincérité est au fond de nous tous.

Je crois aussi qu'on nous a tous donné quelque chose, quelques talents. Il ne faut pas travailler pour soi-même, il faut travailler pour les autres aussi. Quand on travaille pour les autres il y a aussi plus de plaisir car on partage des choses. Croire en ce que l'on fait et partager. Regarder autour de soi. Il faut de l'action, mais pas de stress. Il faut avoir une conscience calme.

Mais, le savez-vous ? Des gens et des artistes émergent, ici en RDC.

Q. Vous voyagez et vous donnez beaucoup.

R. Oui, c'est vrai. Je voyage beaucoup. Tout ce que je gagne je le dépense dans mes déplacements et je donne aussi beaucoup. (Je n'ai pas beaucoup sur mon compte.) Je donne aux jeunes et aux adultes, à la famille. J'aime les enfants de la rue. J'en ai trois que je scolarise. Avant ce n'était rien mais maintenant ils commencent à grandir. Je les nourris, ils viennent boire, ils déposent leurs habits sales... on les lave, on les repasse et puis ils s'en vont. Je suis comme un père pour eux.

Q. S'il n'y avait qu'un seul message à faire passer dans votre œuvre, ce serait lequel ?

R. Je pense que ce serait l'humanité. L'homme triomphe et partage. C'est cela.

\section{NOTES}

1. Quand il n'est pas à l'étranger, Freddy Tsimba travaille à Kinshasa. Récemment, tout près de son atelier, une église du Réveil s'est installée. Le pasteur, qui se nomme le prophète N'Kiere, prêche dans la rue tout près de la parcelle de l'artiste, créant un contraste idéologique comme nous le montre Hippolyte dans son récit graphique «Les enfants de Kinshasa ", in XXI Vingt et un, $\mathrm{n}^{\circ}$ 17, hiver 2012, p. 187-199.

2. Bogumil Jewsiewicki, «Reconnaissance, mémoire culturelle et mise en circulation globale des expériences locales : démarches esthétiques et prises de position éthiques de Sammy Baloji, Steve Bandoma et Freddy Tsimba », Sensus Historiae, vol. XVII, 2014/4, p. 75.

3. Plusieurs œuvres réalisées dans cette période sont reproduites dans le petit livret consacré à Freddy Tsimba par Yoka Lye. Freddy Tsimba. Sculpteur, Les carnets de la Création, éditions de l'œil, 2002.

4. La quatrième de couverture du livret de Lye reproduit cette sculpture ainsi que l'article de Sylvie Chalaye paru dans Africultures du 26 novembre 2003: "L'art peut être subversif tout en étant ludique » entretien de Sylvie Chalaye avec Soly Cissé, Sokey Edorh, Freddy Tsimba et Niko » Africultures: <http://www.africultures.com/php/?nav=article\&no=3224> et son site officiel $<$ https://freddytsimba.wordpress.com/sculptures/\#jp-carousel-229>

5. Voir par exemple son œuvre Échelle de la honte exposée au Botanique à Bruxelles dans le cadre de Yambi en 2007. Voir : <https://www.youtube.com/watch ?v =lgV_XaBM1Fw>

6. Cette œuvre est reproduite dans Roger-Pierre Turine, « Freddy Tsimba », Arts du Congo : d'hier à nos jours. Waterloo, La Renaissance du Livre, 2007, p. 103.

7. Mavambu est la partie de l'arbre où le tronc se scinde en différentes branches, pourrait se traduire par « embranchement». 
8. Joseph-Désiré Mobutu (alias Mobutu Sese Seko Kuku Ngbendu wa Za Banga) a régné de manière autocratique sur la RDC de 1965 à 1990 après avoir mené un coup d'état contre le premier président Kasa-Vubu. Laurent-Désiré Kabila renversa Mobutu et prit le pouvoir en 1997 et le garda jusqu'à son assassinat en 2001. Joseph Kabila lui succéda. En 2006 furent tenues des élections qu'il remporta. En 2011 il fut à nouveau proclamé vainqueur des élections présidentielles.

9. Le 16 février 1992 plusieurs chrétiens furent tués. La Marche des Chrétiens était une manifestation organisée pour la démocratie et contre le pouvoir de Mobutu.

10. L'Académie des Beaux-Arts de Kinshasa existe dans la capitale congolaise depuis 1957. (C'est alors qu'elle avait été transférée de Matadi dans le Bas-Congo. L'École appelée Saint-Luc avait été fondé en 1943 par un missionnaire belge Marc Wallenda.) L'Académie des Beaux-Arts offre des cours au niveau secondaire ( = les humanités artistiques) et au niveau de l'enseignement supérieur : les arts graphiques (architecture d'intérieur et la communication visuelle) et les arts plastiques (céramique, métal battu, peinture et sculpture).

11. Le système scolaire en RDC est copié du système belge. Il consiste en 6 années primaires et 6 années secondaires: les trois premières années du secondaire se nomment cycle inférieur, les trois dernières cycle supérieur. La première année du secondaire est générale.

12. Contrairement aux autres élèves qui avaient commencé les humanités artistiques dès la première année du secondaire, Freddy Tsimba, ayant d'abord fait deux années d'humanités scientifiques, les a rejoints en quatrième secondaire.

13. Belgicisme voulant dire : tableau d'affichage.

14. Une de ces œuvres est reproduite dans l'article d'Aya Kasasa : «Freddy Bienvenue Tsimba: 'Works which are meaningful'« The Courier, Nov. - Dec. 2001, p. 57-58.

<http://ec.europa.eu/development/body/publications/courier/courier189/en/en_057.pdf>

15. Dans son documentaire visible sur YOUTUBE Freddy Tsimba explique qu'il avait dû se faire passer pour un fou pour ramasser les douilles à Kisangani mais que néanmoins il avait été fait prisonnier. Pour prouver qu'il était sculpteur il avait dû faire 200 marmites : <https:// www.youtube.com/watch ?v=BM8fuDeqNOE>. Voir également Vincent Lombume Kalimasi (textes) et Cédrick Nzolo Ngamobu (photographies), Freddy Tsimba. Légendes et saisons de métal, Bruxelles, Le Cri édition, 2012, p. 8.

16. Ses œuvres portent presque toutes le titre de Silhouette.

17. Tsimba dit se souvenir que la première machette qu'il a vue enfant fut fabriquée en Chine (Jewsiewicki op. cit.p. 83).

18. Dans le livre de Vincent Lombume Kalimasi (textes) et Cédrick Nzolo Ngamobu (photographies) op.cit. on trouve des reproductions de ce «cortège ». Le film documentaire Kinshasa Mboka Té réalisé par Douglas Ntimasiemi et Raffi Aghekian montre la fabrication de la maison machettes et puis les réactions du public quand elle fut exposée au marché Liberté de la commune Massina.

19. < http://www.jeuneafrique.com/Article/ARTJAWEB20121112121019/benin-cotonou-artcontemporain-genocide-rwandaisbenin-abomey-royaume-de-l-art-contemporain.html>

20. < http://www.lesmediasducitoyen.cd/actus/freddy-tsimba-exporte-son-savoir-faire-aumaroc> L'œuvre sera montrée à la biennale de Casablanca l'année prochaine.

21. Grâce à ses dons de diplomate, Léopold II se fit accepter à la Conférence de Berlin (15 nov. 1884 - 26 févr. 1885) roi d'un territoire quatre-vingt fois la taille de la Belgique, par les grandes puissances de l'époque. Il régna sur l'État Indépendant du Congo (É.I.C.) de 1885 à 1908 et y appliqua un régime de travail forcé. Il avait une résidence importante à Ostende.

22. Voir article « Espace Schengen : Flux migratoires et Directive retour. L'expulsion de Freddy Tsimba le 18 octobre 2011 », Africultures (publié 19/10/2011) <http://www.africultures.com/php/ index.php?nav=article\&no=10443>. 
23. Voir « Un murmure radiophonique sur l'expulsion de Freddy Tsimba » Africultures (publié en octobre 2011

$<$ http://www.africultures.com/php/index.php/index.php?

nav=murmure \&no=8014>

24. Voir l'image 8 du site du vernissage de Freddy Tsimba à L'institut Français de Kinshasa: <http://www.institutfrancais-kinshasa.org/index.php/fre/Institut-Francais-en-images/

Vernissage-expo-9-6-de-Freddy-Tsimba>

\section{AUTEUR}

\section{THÉRÈSE DE RAEDT}

Associate professor, section française du département de langues et littérature à l'université de Utah à Salt Lake City.

Les thématiques qu'elle aborde dans ses publications se concentrent sur trois axes de recherche : les représentations de l'Africain dans la littérature et les arts plastiques en France jusqu'à l'abolition de l'esclavage, les lieux de villégiature envisagés comme de nouveaux espaces postcoloniaux, et les liens interculturels établis volontairement ou par la force dans le monde francophone. 